\title{
Symmetric Identities from an Invariant in Partition Conjugation and Their Applications in $q$-Series
}

\author{
Sandy H. L. Chen 1,2 \\ ${ }^{1}$ School of Science, Tianjin Chengjian University, Tianjin, China \\ ${ }^{2}$ Center for Combinatorics, LPMC-TJKLC, Nankai University, Tianjin, China \\ Email: chenhuanlin@mail.nankai.edu.cn
}

Received 21 January 2014; revised 19 February 2014; 17 March 2014

Copyright (C) 2014 by authors and Scientific Research Publishing Inc.

This work is licensed under the Creative Commons Attribution International License (CC BY).

http://creativecommons.org/licenses/by/4.0/

\section{(c) (i) Open Access}

\begin{abstract}
For every partition $\lambda$ and its conjugation $\lambda^{\prime}$, there is an important invariant $I_{d}(\lambda)$, which denotes the number of different parts. That is, $I_{d}(\lambda)=l_{d}\left(\lambda^{\prime}\right)$. We will derive a series of symmetric $q$-identities from the invariant in partition conjugation by studying modified Durfee rectangles. The extensive applications of the several symmetric $q$-identities in $q$-series [1] will also be discussed. Without too much effort one can obtain much well-known knowledge as well as new formulas by proper substitutions and elementary calculations, such as symmetric identities, mock theta functions, a two-variable reciprocity theorem, identities from Ramanujan's Lost Notebook and so on.
\end{abstract}

Keywords

Integer Partitions, Conjugation, Invariant, $q$-Series, Symmetric Identities

\section{Definitions and Combinatorial Interpretations}

We shall first present some basic definitions and combinatorial interpretations for basic hypergeometric series and integer partition. For simplicity, unless stated otherwise we shall assume that $n$ is a nonnegative integer and $|q|<1$, let

$$
(a ; q)_{\infty}=(1-a)(1-a q) \cdots .
$$

Definition 1.1 For any integer $n$, the $q$-shifted factorial is defined by 


$$
(a ; q)_{n}=\frac{(a ; q)_{\infty}}{\left(a q^{n} ; q\right)_{\infty}} .
$$

Definition 1.2 A partition $\lambda$ of a positive integer $n$ is a finite nonincreasing sequence of integers $\lambda_{1}, \lambda_{2}, \cdots, \lambda_{r}$, such that $\sum_{i=1}^{r} \lambda_{i}=n . \quad \lambda^{\prime}$ is the conjugate partition of $\lambda$. The largest part, the number of parts, as well as the sum of the parts are denoted by $a(\lambda), l(\lambda)$ and $|\lambda|$, respectively.

An effective device for studying partitions is the graphical representation. For a partition $\lambda$, its $m \times n$ Durfee rectangle is the maximum rectangle contained in the Ferrers diagram of $\lambda$. Conjugation and the several invariants have been used in a variety of ways over the years, see Andrews's encyclopedia [2]. It is worth pointing out that there is a fundamental invariant which despite its simplicity has not received too much attention. This is $l_{d}(\lambda)$, the number of different parts of $\lambda$. For all partitions $\lambda$ and its conjugation $\lambda^{\prime}$,

$$
l_{d}(\lambda)=l_{d}\left(\lambda^{\prime}\right) .
$$

In this paper, we shall show how (1) could be used to obtain a series of symmetric identities by studying modified Durfee rectangles. Consider the expansion

$$
\frac{1-a b q^{i}}{1-b q^{i}}=\frac{1-b q^{i}+b q^{i}-a b q^{i}}{1-b q^{i}}=1+(1-a)\left(b q^{i}+b^{2} q^{2 i}+b^{3} q^{3 i}+\cdots\right) .
$$

We interpret this as an expansion involving only one part, namely $i$, where the power of $b$ records $l(\lambda)$, while that of $(1-a)$ indicates whether the part occurs or not. Thus, we interpret

$$
\frac{(a b q)_{n}}{(b q)_{n}}=\sum_{a(\lambda) \leq n}(1-a)^{l_{d}(\lambda)} b^{l(\lambda)} q^{|\lambda|}
$$

as the generating function of partitions $\lambda$ into parts less than or equal to $n$, such that the power of $b$ records $l(\lambda)$, while that of $(1-a)$ indicates $l_{d}(\lambda)$. Then it follows that the three-parameter generating function for all unrestricted partitions $\lambda$, namely, the function

$$
G(a, b, c ; q)=\sum_{\lambda}(1-a)^{l_{d}(\lambda)} b^{l(\lambda)} c^{a(\lambda)} q^{|\lambda|}=1+\sum_{n=1}^{\infty} \frac{(1-a)(a b q)_{n-1} b c^{n} q^{n}}{(b q)_{n}} .
$$

We consider all partitions $\lambda$ for which $a(\lambda)=n$. This accounts for the term $c^{n} q^{n}$ in (3). Since $\lambda$ contains $n$ as a part, we have the factors $(1-a)$ and $b$ in the numerator. The part $n$ may repeat, which is given by $\frac{1}{1-b q^{n}}$. The repetition of $n$ will not contribute to $l_{d}(\lambda)$ and so there is no further power of $(1-a)$ contributed by the part $n$. The part $1,2, \cdots, n-1$ could repeat and their contribution to the generating function is given by the term

$$
\frac{(a b q)_{n-1}}{(b q)_{n-1}}
$$

Formula (3) follows.

\section{Symmetric Expressions for $G(a, b, c ; q)$}

In this section, we give several symmetric expansions for $G(a, b, c ; q)$ via modified Durfee rectangles analysis of partition.

Theorem 2.1

$$
G(a, b, c ; q)=1+\frac{b c q(1-a)}{1-c q}+\sum_{n=1}^{\infty} \frac{(1-a) b^{n+1} c^{n} q^{n^{2}+n}(a b q)_{n-1}(a c q)_{n}\left(1-a b c q^{2 n+1}\right)}{(b q)_{n}(c q)_{n+1}} .
$$

Proof. For every partitions $\lambda$ and $n \geq 1$, the Ferrers graph contains a largest $n \times(n+1)$ Durfee rectangle with side $n$ horizontally by $n+1$ vertically. Then to the right of the Durfee rectangle, we have a partition $\lambda_{a}$ 
which has at most $n+1$ parts, or equivalently, $\lambda_{a}^{\prime}$, the conjugate of $\lambda_{a}$, with largest part $\leq n+1$. Below the Durfee rectangle we have a partition $\lambda_{b}$ whose parts $\leq n$. We now divide our consideration into four cases.

1) $l\left(\lambda_{a}\right)=n+1, a\left(\lambda_{b}\right) \leq n-1$

We consider the contribution of the partition $\lambda_{a}$ to $G(a, b, c ; q)$ by utilizing its conjugate $\lambda_{a}^{\prime}$.

$$
\sum_{\lambda_{a}}(1-a)^{l_{d}\left(\lambda_{a}\right)} c^{a\left(\lambda_{a}\right)} q^{\left|\lambda_{a}\right|}=\sum_{\lambda_{a}^{\prime}}(1-a)^{l_{d}\left(\lambda_{a}^{\prime}\right)} c^{l\left(\lambda_{a}^{\prime}\right)} q^{\left|\lambda_{a}^{\prime}\right|}=\frac{(1-a) c q^{n+1}}{1-c q^{n+1}} \frac{(a c q)_{n}}{(c q)_{n}}=\frac{(1-a) c q^{n+1}(a c q)_{n}}{(c q)_{n+1}} .
$$

The factor $c q^{n+1}$ in the numerator arises from the column of length $n+1$ lying to the right of the Durfee rectangle. Because $\lambda_{a}$ contributes nothing to $l(\lambda)$, we omit $b$.

The contribution of the partition $\lambda_{b}$ to $G(a, b, c ; q)$ is

$$
\sum_{\lambda_{b}}(1-a)^{l_{d}\left(\lambda_{b}\right)} b^{a\left(\lambda_{b}\right)} q^{\left|\lambda_{b}\right|}=\frac{(a b q)_{n-1}}{(b q)_{n-1}} .
$$

Note that the parameter $c$ is absent because the partition $\lambda_{b}$ has no contribution to $a(\lambda)$.

Meanwhile, the contribution of the modified Durfee rectangle to $G(a, b, c ; q)$ is

$$
b^{n+1} c^{n} q^{n(n+1)} \text {. }
$$

Thus, we derive the generating function of every unrestricted partitions $\lambda$ :

$$
\frac{(1-a) b^{n+1} c^{n+1} q^{n^{2}+2 n+1}(a b q)_{n-1}(a c q)_{n}}{(b q)_{n-1}(c q)_{n+1}} .
$$

2) $l\left(\lambda_{a}\right) \leq n, a\left(\lambda_{b}\right)=n$.

The generating function of every unrestricted partitions $\lambda$ :

$$
\frac{(1-a) b^{n+2} c^{n} q^{n^{2}+2 n}(a b q)_{n-1}(a c q)_{n}}{(b q)_{n}(c q)_{n}} .
$$

3) $l\left(\lambda_{a}\right) \leq n, a\left(\lambda_{b}\right) \leq n-1$.

The generating function of every unrestricted partitions $\lambda$ :

$$
\frac{(1-a) b^{n+1} c^{n} q^{n^{2}+n}(a b q)_{n-1}(a c q)_{n}}{(b q)_{n-1}(c q)_{n}} .
$$

4) $l\left(\lambda_{a}\right)=n+1, a\left(\lambda_{b}\right)=n$.

The generating function of every unrestricted partitions $\lambda$ :

$$
\frac{(1-a)^{2} b^{n+2} c^{n+1} q^{n^{2}+3 n+1}(a b q)_{n-1}(a c q)_{n}}{(b q)_{n}(c q)_{n+1}} .
$$

Summing these four generating functions for $n \geq 1$, we get an expression for $G(a, b, c ; q)$ :

$$
G(a, b, c ; q)=1+\frac{b c q(1-a)}{1-c q}+\sum_{n=1}^{\infty} \frac{(1-a) b^{n+1} c^{n} q^{n^{2}+n}(a b q)_{n-1}(a c q)_{n}\left(1-a b c q^{2 n+1}\right)}{(b q)_{n}(c q)_{n+1}} .
$$

Remark 2.2 Under partition conjugation, $l(\lambda)$ and $a(\lambda)$ are interchanged, it follows that $b$ and $c$ are symmetric in $G(a, b, c ; q)$.

Theorem 2.3 From formula (3) and the symmetry of $b$ and $c$, we have

$$
\begin{aligned}
G(a, b, c ; q) & =1+\sum_{n=1}^{\infty} \frac{(1-a)(a b q)_{n-1} b c^{n} q^{n}}{(b q)_{n}} \\
& =1+\sum_{n=1}^{\infty} \frac{(1-a)(a c q)_{n-1} c b^{n} q^{n}}{(c q)_{n}}
\end{aligned}
$$


Theorem 2.4 From Theorem 2.1 and the symmetry of $b$ and $c$, we have

$$
\begin{aligned}
G(a, b, c ; q)= & 1+\frac{b c q(1-a)}{1-c q}+\sum_{n=1}^{\infty} \frac{(1-a) b^{n+1} c^{n} q^{n^{2}+n}(a b q)_{n-1}(a c q)_{n}\left(1-a b c q^{2 n+1}\right)}{(b q)_{n}(c q)_{n+1}} \\
& =1+\frac{b c q(1-a)}{1-b q}+\sum_{n=1}^{\infty} \frac{(1-a) b^{n} c^{n+1} q^{n^{2}+n}(a b q)_{n}(a c q)_{n-1}\left(1-a b c q^{2 n+1}\right)}{(b q)_{n+1}(c q)_{n}} \\
& =1+\sum_{n=1}^{\infty} \frac{(1-a) b^{n} c^{n} q^{n^{2}}(a b q)_{n-1}(a c q)_{n-1}\left(1-a b c q^{2 n}\right)}{(b q)_{n}(c q)_{n}},
\end{aligned}
$$

where (8) results from the $n \times n \quad$ Durfee square analysis.

\section{The Applications of the Symmetric Identities in $q$-Series}

In this section, we shall explore the extensive applications of formulas (4) to (8) in $q$-series. Without too much effort one can obtain much well-know knowledge as well as new formulas by proper substitutions and elementary calculations. It will be overly clear that the list of nice application is sheer endless.

\subsection{Symmetric Identities}

From (4) and (5), we get the following beautiful symmetric identity.

Corollary 3.1

$$
\sum_{n=0}^{\infty} \frac{(a b q)_{n}}{(b q)_{n+1}}(c q)^{n}=\sum_{n=0}^{\infty} \frac{(a c q)_{n}}{(c q)_{n+1}}(b q)^{n} .
$$

Taking $a=q / a b c d, b=a c / q, c=b c / q$ in (9), we derive the following identity, from which Liu [3] proved an identity of Andrews.

Corollary 3.2

$$
(1-b c) \sum_{n=0}^{\infty} \frac{(q / b d)_{n}}{(a c q)_{n}}(b c)^{n}=(1-a c) \sum_{n=0}^{\infty} \frac{(q / a d)_{n}}{(b c q)_{n}}(a c)^{n} .
$$

Setting $q \rightarrow q^{2}$ and then taking $a=a q / b, c=t$ in (4) and (5), we have

Corollary 3.3

$$
\sum_{n=0}^{\infty} \frac{\left(a q^{3} ; q^{2}\right)_{n}}{\left(b q^{2} ; q^{2}\right)_{n+1}} b t^{n+1} q^{2 n+2}=\sum_{n=0}^{\infty} \frac{\left(\frac{a t q^{3}}{b} ; q^{2}\right)_{n}}{\left(t q^{2} ; q^{2}\right)_{n+1}} t b^{n+1} q^{2 n+2},
$$

which was first stated and proved by N. J. Fine [4]. Andrews derived it combinatorially from the consideration of partitions without repeated odd parts in [5].

\subsection{Mock Theta Functions}

In his famous last letter to Hardy [6], Ramanujan introduced 17 mock theta functions without giving an explicit definition, among which, one third order mock theta function is as follows

$$
f(q)=\sum_{n=0}^{\infty} \frac{q^{n^{2}}}{(-q)_{n}^{2}} .
$$

In 1966, Andrews [7] defined the following generalization of $f(q)$

$$
f(\alpha ; q)=\sum_{n=0}^{\infty} \frac{\alpha^{n} q^{n^{2}-n}}{(-q)_{n}(-\alpha)_{n}} .
$$


Moreover, Watson [8] added three functions to the list of Ramanujan's third order mock theta functions and the following identity is just one of them

$$
\omega(q)=\sum_{n=0}^{\infty} \frac{q^{2 n(n+1)}}{\left(q ; q^{2}\right)_{n+1}^{2}} .
$$

By proper substitutions in Theorem 2.3 and 2.4, we obtain much simpler expressions for the above mock theta functions. Through the specializations $a=0, b=-1$ and $c=-\alpha q^{-1}$ in (4) and (8), we derive a simpler transformation formula for $f(\alpha ; q)$ :

Corollary 3.4

$$
\sum_{n=0}^{\infty} \frac{\alpha^{n} q^{n^{2}-n}}{(-q)_{n}(-\alpha)_{n}}=2-\sum_{n=0}^{\infty} \frac{(-1)^{n} \alpha^{n}}{(-q)_{n}} .
$$

Taking $\alpha=q$ in (14), a representation for $f(q)$ follows, with the powers diminished

$$
\sum_{n=0}^{\infty} \frac{q^{n^{2}}}{(-q)_{n}^{2}}=2-\sum_{n=0}^{\infty} \frac{(-1)^{n} q^{n}}{(-q)_{n}} .
$$

Fine [1] first derived (15) by applying some transformation formulas and Liu [[9], Theorem 3.7] proved it combinatorially by an application of involution. Changing $q$ to $q^{2}$ and then putting $a=0, b=q, c=1 / q$ in (4) and (6), we get a new expression for $\omega(q)$, with the powers diminished:

Corollary 3.5

$$
\sum_{n=0}^{\infty} \frac{q^{2 n(n+1)}}{\left(q ; q^{2}\right)_{n+1}^{2}}=\sum_{n=0}^{\infty} \frac{q^{n}}{\left(q ; q^{2}\right)_{n+1}} .
$$

\subsection{A Two-Variable Reciprocity Theorem}

Taking $a=-1 / b c, b=-a$ and then letting $c \rightarrow 0$ in (4), we have

$$
\rho(a, b)=\left(1+\frac{1}{b}\right) \sum_{n=0}^{\infty} \frac{(-1)^{n} q^{n(n+1) / 2} a^{n} b^{-n}}{(-a q)_{n}} .
$$

In his lost notebook [10], Ramanujan offers a beautiful reciprocity theorem

$$
\rho(a, b)-\rho(b, a)=\left(\frac{1}{b}-\frac{1}{a}\right) \frac{(a q / b)_{\infty}(b q / a)_{\infty}(q)_{\infty}}{(-a q)_{\infty}(-b q)_{\infty}} .
$$

After the same substitutions in (5) and (7), respectively, we get

\section{Corollary 3.6}

$$
\begin{gathered}
\rho(a, b)=1+\frac{1}{b} \sum_{n=0}^{\infty}\left(-\frac{1}{b}\right)_{n}(-a q)^{n} \\
=1+\sum_{n=0}^{\infty} \frac{(-1 / b)_{n} a^{2 n} b^{-n-1} q^{3 n(n+1) / 2}\left(1-a q^{2 n+1} / b\right)}{(-a q)_{n+1}} .
\end{gathered}
$$

Formula (18) is a slightly simpler representation of $\rho(a, b)$. From (19) and the above reciprocity theorem (17), we get the following two variable generalization of the Quintuple Product Identity [[11], Theorem 3.1] without any proof:

Corollary 3.7 A Two-Variable Generalization of the Quintuple Product Identity

For $a, b \neq q^{-n}, 1 \leq n<\infty$, 


$$
\begin{aligned}
& \left(\frac{1}{a}-\frac{1}{b}\right) \frac{(a q / b)_{\infty}(b q / a)_{\infty}(q)_{\infty}}{(a q)_{\infty}(b q)_{\infty}} \\
& =\sum_{n=0}^{\infty} \frac{(-1)^{n}(1 / a)_{n} a^{-n-1} b^{2 n} q^{3 n(n+1) / 2}\left(1-b q^{2 n+1} / a\right)}{(b q)_{n+1}}-\sum_{n=0}^{\infty} \frac{(-1)^{n}(1 / b)_{n} a^{2 n} b^{-n-1} q^{3 n(n+1) / 2}\left(1-a q^{2 n+1} / b\right)}{(a q)_{n+1}} .
\end{aligned}
$$

\subsection{Identities from Ramanujan's Lost Notebook}

By special substitutions, we could go through a series of important Entries in Ramanujan's Lost Notebook [12]. We take several of them as examples, for their combinatorial proofs, see [13]. The function

$$
\phi(a)=\sum_{n=0}^{\infty} \frac{a^{n} q^{n(n+1) / 2}}{(b q)_{n}},
$$

is defined by Ramanujan. Setting $a=-a / b c, b=b$, and then letting $c \rightarrow 0$, then (4) and (6) can be reduced to

Corollary 3.8 (Entry 9.2.2)

$$
\phi(a)=\sum_{n=0}^{\infty} \frac{(-a q / b)_{n} a^{n} b^{n} q^{n(3 n+1) / 2}\left(1+a q^{2 n+1}\right)}{(b q)_{n}} .
$$

The same substitutions in (4) and (8), we have

Corollary 3.9 (Entry 9.2.3)

$$
\phi(a)=\sum_{n=0}^{\infty} \frac{(-a q / b)_{n-1} a^{n} b^{n-1} q^{n(3 n-1) / 2}\left(1+a q^{2 n}\right)}{(b q)_{n}} .
$$

Putting $a=b, b=-a$ and $c=a$, and then setting $b \rightarrow 0$ in (4) and (8), we have

Corollary 3.10 (Entry 9.2.4)

$$
\sum_{n=0}^{\infty} \frac{(-1)^{n} a^{2 n} q^{n^{2}}}{\left(a^{2} q^{2} ; q^{2}\right)_{n}}=1-a \sum_{n=1}^{\infty} \frac{a^{n} q^{n}}{(-a q)_{n}} .
$$

For the above identity, it is interesting to note that the terms in $a$ and $q$ on the right side are the same as those on the left side, but with the powers diminished. In (4) and (6), we replace $q$ by $q^{2}$ and take $a=b, b=a q$ and $c=-a / q$, and then set $b \rightarrow 0$, the Entry 9.2.5 in Ramanujan's Lost Notebook [12] follows:

Corollary 3.11 (Entry 9.2.5)

$$
\sum_{n=0}^{\infty} \frac{(-1)^{n} a^{n} q^{n}}{\left(a q ; q^{2}\right)_{n+1}}=\sum_{n=0}^{\infty} \frac{(-1)^{n} a^{2 n} q^{2 n^{2}+2 n}}{\left(a^{2} q^{2} ; q^{4}\right)_{n+1}} .
$$

Berndt and Yee [13] proved the above two corollaries combinatorially by accounting for partitions into distinct parts. Replacing $q$ by $q^{2}$ and taking $a=q, b=a$ and $c=a / q$ in (4) and (6), then we get the following Entry. Berndt and Yee [13] derived it by employing 2 -modular partitions.

Corollary 3.12 ( Entry 9.3.1)

$$
\sum_{n=0}^{\infty} \frac{\left(a q ; q^{2}\right)_{n}(a q)^{n}}{\left(a q^{2} ; q^{2}\right)_{n}}=\sum_{n=0}^{\infty} a^{2 n} q^{2 n^{2}+n}\left(1+a q^{2 n+1}\right)=\sum_{n=0}^{\infty} a^{n} q^{n(n+1) / 2} .
$$

In (4) and (6), we take $a=-a / c, b=-a$, and then set $c \rightarrow 0$, Entry 9.4.1 follows:

\section{Corollary 3.13 (Entry 9.4.1)}

$$
\sum_{n=0}^{\infty} \frac{(-1)^{n} a^{2 n} q^{n(n+1) / 2}}{(-a q ; q)_{n}}=\sum_{n=0}^{\infty} a^{3 n} q^{n(3 n+1) / 2}\left(1-a^{2} q^{2 n+1}\right) .
$$

This identity was derived from Franklin involution by Berndt and Yee [13] and was also got from two entries 
by Warnaar [14], where analytic methods were employed.

\subsection{Further Consequences}

\section{Corollary 3.14}

$$
\sum_{n=0}^{\infty} \frac{q^{n}}{\left(-q ; q^{2}\right)_{n+1}}=\sum_{n=0}^{\infty}(-1)^{n} q^{6 n^{2}+4 n}\left(1+q^{4 n+2}\right) .
$$

Proof. Taking $q \rightarrow q^{2}$, and then letting $a=0, b=-1 / q$ and $c=1 / q$ in (4) and (8), we have

$$
\begin{aligned}
& \sum_{n=0}^{\infty} \frac{q^{n}}{\left(-q ; q^{2}\right)_{n+1}}=-\sum_{n=1}^{\infty} \frac{(-1)^{n} q^{2 n^{2}-2 n}}{\left(q^{2} ; q^{4}\right)_{n}} \\
& =1-\sum_{n=0}^{\infty}(-1)^{n} q^{6 n^{2}-4 n}\left(1-q^{8 n}\right) \quad \text { (by) }=\sum_{n=0}^{\infty}(-1)^{n} q^{6 n^{2}+4 n}\left(1+q^{4 n+2}\right) .
\end{aligned}
$$

Identity (22) is a false theta series identity. Results like these were studied by L. J. Rogers [15], however, the elegant result appears to have escaped him. Andrews [16] proved identity (22) by using three transformation formulas and showed that (22) implied a partition identity like that deduced from Euler's Pentagonal Number Theorem ([2], p. 10).

Taking $a=0, b=-1$ and $c=1$ in (4) and (5), we generalize the not at all deep but elegant identity:

\section{Corollary 3.15}

$$
\sum_{n=0}^{\infty} \frac{q^{n}}{(-q ; q)_{n}}=2-\sum_{n=0}^{\infty} \frac{(-1)^{n} q^{n}}{(q ; q)_{n}}=2-\frac{1}{(-q ; q)_{\infty}} .
$$

Taking $a=-a, b=\frac{1}{\sqrt{a}}, c=\frac{1}{\sqrt{a}}$, and then setting $a \rightarrow \infty$, (4) and (8) can be reduced to the famous Gauss triangle series

\section{Corollary 3.16}

$$
1+\sum_{n=1}^{\infty} q^{2 n^{2}-n}\left(1+q^{2 n}\right)=\sum_{n=-\infty}^{\infty} q^{2 n^{2}-n}=\sum_{n=0}^{\infty} q^{n(n+1) / 2}
$$

\section{References}

[1] Gasper, G. and Rahman, M. (2004) Basic Hypergeometric Series. 2nd Edition, Cambridge University Press, Cambridge.

[2] Andrews, G.E. (1976) The Theory of Partitions, Encyclopedia of Math, and Its Applications. Addison-Wesley Publishing Co., Boston.

[3] Liu, Z.G. (2003) Some Operator Identities and Q-Series Transformation Formulas. Discrete Mathematics, 265, 119139. http://dx.doi.org/10.1016/S0012-365X(02)00626-X.

[4] Fine, N.J. (1988) Basic Hypergeometric Series and Applications. Mathematical Surveys and Monographs, 1988. http://dx.doi.org/10.1090/surv/027

[5] Andrews, G.E. (1972) Two Theorems of Gauss and Allied Identities Proved Arithmetically. Pacific Journal of Mathematics, 41, 563-578. http://dx.doi.org/10.2140/pjm.1972.41.563.

[6] Berndt, B.C. and Rankin, R.A. (1995) Ramanujan: Letters and Commentary. American Mathematical Society, Providence, London Mathematical Society, London.

[7] Andrews, G.E. (1966) On Basic Hypergeometric Series, Mock Theta Functions, and Partitions (I). The Quarterly Journal of Mathematics, 17, 64-80. http://dx.doi.org/10.1093/qmath/17.1.64.

[8] Watson, G.N. (1936) The Final Problem: An Account of the Mock Theta Functions. Journal of the London Mathematical Society, 11, 55-80. http://dx.doi.org/10.1112/jlms/s1-11.1.55

[9] Liu, X.C. (2012) On Flushed Partitions and Concave Compositions. European Journal of Combinatorics, 33, $663-678$. http://dx.doi.org/10.1016/j.ejc.2011.12.004 
[10] Ramanujan, S. (1988) The Lost Notebook and Other Unpublished Paper. Springer-Verlag, Berlin.

[11] Berndt, B.C., Chan, S.H., Yeap, B.P. and Yee, A.J. (2007) A Reciprocity Theorem for Certain Q-Series Found in Ramanujan's Lost Notebook. The Ramanujan Journal, 13, 27-37. http://dx.doi.org/10.1007/s11139-006-0241-5

[12] Andrews, G.E. and Berndt, B.C. (2005) Ramanujan’s Lost Notebook, Part I. Springer, New York.

[13] Berndt, B.C. and Yee, A.J. (2003) Combinatorial Proofs of Identities in Ramanujan's Lost Notebook Associated with the Rogers-Fine Identity and False Theta Functions. Annals of Combinatorics, 7, 409-423. http://dx.doi.org/10.1007/s00026-003-0194-y.

[14] Warnaar, S.O. (2003) Partial Theta Functions. I. Beyond the Lost Notebook. Proceedings of the London Mathematical Society, 87, 363-395. http://dx.doi.org/10.1112/S002461150201403X.

[15] Rogers, L.J. (1917) On Two Theorems of Combinatory Analysis and Some Allied Identities. Proceedings of the London Mathematical Society, 16, 316-336.

[16] Andrews, G.E. (1979) An Introduction to Ramanujan's Lost Notebook, The American Mathematical Monthly, 86, 89-108. http://dx.doi.org/10.2307/2321943 